\title{
Finite Element Heterogeneous \\ Multiscale Method \\ for the Wave Equation
}

\author{
Assyr Abdulle, Marcus J. Grote
}

Department of Mathematics

Preprint No. 2010-01

University of Basel

July 10

Rheinsprung 21

CH - 4051 Basel

Switzerland

www.math.unibas.ch 


\title{
FINITE ELEMENT HETEROGENEOUS MULTISCALE METHOD FOR THE WAVE EQUATION
}

\author{
ASSYR ABDULLE* AND MARCUS J. GROTE ${ }^{\dagger}$
}

\begin{abstract}
A finite element heterogeneous multiscale method (FE-HMM) is proposed for the wave equation with highly oscillatory coefficients. It is based on a finite element discretization of an effective wave equation at the macro scale, whose a priori unknown effective coefficients are computed on sampling domains at the micro scale within each macro finite element. Hence the computational work involved is independent of the highly heterogeneous nature of the medium at the smallest scale. Optimal error estimates in the energy norm and the $L^{2}$ norm and convergence to the homogenized solution are proved, when both the macro and the micro scale are refined simultaneously. Numerical experiments corroborate the theoretical convergence rates and illustrate the behavior of the numerical method on periodic and heterogeneous media.
\end{abstract}

Key words. multiscale method, heterogeneous media, numerical homogenization, a priori error analysis, wave equation, second-order hyperbolic problems

AMS subject classifications. 65N30,65M60,74Q10,74Q15,74Q20

1. Introduction. Wave phenomena from a wide range of applications, such as seismic inversion, medical imaging and therapy, or the design of multiphase composite materials are modeled by the wave equation with highly oscillatory coefficients. Yet when waves propagate across inhomogeneous media with microscopic heterogeneities, assumed to occur at a scale larger than atomistic, standard numerical methods, such as finite element methods (FEM) or finite difference (FD) methods, become inefficient. As classical numerical schemes require grid resolution of the medium downto its finest scales, they typically lead to extremely large problem sizes although the scales of interest, such as the wavelength, often occur at a macroscopic level,

Here we consider wave equations with highly oscillatory velocity fields, which vary at a scale $\varepsilon$ much smaller than the scales of interest. For periodic or random stationary fields, the analytic treatment of such problems usually relies on homogenization theory $[11,13,17]$, where the highly oscillatory velocity field $a_{\varepsilon}$ is replaced by a properly averaged (homogenized) field $a_{0}$ that captures the essential macroscopic features in the limit $\epsilon \rightarrow 0$. Unfortunately, explicit formulas for $a_{0}$ are rarely available so that numerical methods that circumvent those restrictions are needed.

Numerical methods for elliptic and parabolic homogenization problems have been studied in many papers and various approaches have been proposed - see $[2,6,8]$ and the references therein. In contrast, the literature for the numerical homogenization of wave equations is rather scarce. Owhadi and Zhang [30] proposed a numerical method for the wave equation in the spirit of the so-called multiscale finite element method of Hou et al. [25]. Their method is designed for strongly non-local media and relies on a global change of coordinates map to transform coarse scale FE basis functions into multiscale FE basis functions. Although quite general, that approach requires the numerical approximation of the change of coordinates map, which is far from trivial and rather expensive. Recently, Engquist, Holst and Runborg [18] proposed a FD method in the framework of heterogenous multiscale methods (HMM) [19].

Here we propose a multiscale FEM for the numerical homogenization of the wave

\footnotetext{
*Mathematics Section, École Polytechnique Fédérale de Lausanne, Switzerland, assyr.abdulle@epfl.ch

$\dagger$ Institute of Mathematics, University of Basel, marcus.grote@unibas.ch
} 
equation with heterogenous coefficients. Our method differs from [30], as it is based on local micro computations within a macroscopic FE mesh. Thus scale separation (e.g. random stationarity) is needed for our method to be effective. In turn we achieve a significant reduction in the computational work, when scale separation can be assumed. Our method also differs from [18], as it based on a FE discretization. Moreover, we derive fully discrete optimal error estimates in the energy and in the $L^{2}$ norm. Such results constitute to the best of our knowledge the first fully discrete error analysis (in space) for the numerical homogenization of the wave equation.

Our paper is organized as follows. In section 2 we introduce the homogenization problem for the wave equation and recall some known analytical results. In section 3 we introduce our FE-HMM for the wave equation. The analysis of the numerical method is given in section 4. Various numerical experiments are given in section 5 to test the behavior of the numerical method and verify the theoretical convergence results.

Notations. Let $\Omega \subset \mathbb{R}^{d}$ be open and denote by $W^{s, p}(\Omega)$ the standard Sobolev space. For $p=2$ we use the notation $H^{s}(\Omega)$ and $H_{0}^{1}(\Omega)$, and denote by $W_{p e r}^{1}(Y)=\{v \in$ $\left.H_{\text {per }}^{1}(Y) ; \int_{Y} v d x=0\right\}$, where $H_{\text {per }}^{s}(Y)$ is defined as the closure of $\mathcal{C}_{p e r}^{\infty}(Y)$ (the subset of $\mathcal{C}^{\infty}\left(\mathbb{R}^{d}\right)$ of periodic functions in the unit cube $\left.Y=\right] 0,1\left[^{d}\right)$ with respect to the $H^{s}$ norm. For a domain $D \subset \Omega,|D|$ denotes the measure of $D$. The derivatives $\frac{\partial}{\partial t} \frac{\partial^{2}}{\partial t^{2}}, \ldots$ are sometimes written as $\partial_{t}, \partial_{t t}, \ldots$ or alternatively as $\partial_{t^{k}}$. For $T>0$ and $B$ a Banach space with norm $\|\cdot\|_{B}$, we denote by $L^{p}(0, T ; B)=L^{p}(B), 1 \leq p \leq \infty$ the Bochner space of functions $v:(0, T) \rightarrow B$. Equipped with the norm

$$
\|v\|_{L^{p}(0, T ; B)}=\left(\int_{0}^{T}\|v(t)\|_{B}^{p} d t\right)^{\frac{1}{p}}
$$

the space $L^{p}(0, T ; B)$ is also a Banach space - see [21] for details.

2. Model Problem. We consider the wave equation

$$
\begin{aligned}
& \left.\partial_{t t} u_{\varepsilon}-\nabla \cdot\left(a^{\varepsilon} \nabla u_{\varepsilon}\right)=F \text { in } \Omega \times\right] 0, T[ \\
& \left.u_{\varepsilon}=0 \text { on }\right] 0, T[\times \partial \Omega \\
& u_{\varepsilon}(x, 0)=f(x), \quad \partial_{t} u_{\varepsilon}(x, 0)=g(x) \quad \text { in } \Omega,
\end{aligned}
$$

where $a^{\varepsilon}$ is symmetric, satisfies $a^{\varepsilon}(x) \in\left(L^{\infty}(\Omega)\right)^{d \times d}$ and is uniformly elliptic and bounded, i.e.,

$$
\exists \lambda, \Lambda>0 \text { such that } \lambda|\xi|^{2} \leq a^{\varepsilon}(x) \xi \cdot \xi \leq \Lambda|\xi|^{2}, \forall \xi \in \mathbb{R}^{d} \text { and } \forall \varepsilon .
$$

Here $\varepsilon$ represents a small scale in the problem, which characterizes the multiscale nature of the tensor $a^{\varepsilon}(x)$. Although we choose Dirichlet boundary conditions here, for simplicity, the analysis below easily generalizes to other types of boundary conditions.

We make the following standard regularity assumptions:

$$
\begin{aligned}
& F \in L^{2}\left(0, T ; L^{2}(\Omega)\right), \\
& g \in H_{0}^{1}(\Omega), \\
& f \in L^{2}(\Omega),
\end{aligned}
$$

and let $E$ denote the Hilbert space,

$$
E=\left\{v ; v \in L^{2}\left(0, T ; H_{0}^{1}(\Omega)\right), v^{\prime} \in L^{2}\left(0, T ; L^{2}(\Omega)\right)\right\},
$$


equipped with the norm

$$
\|v\|_{E}=\|v\|_{L^{2}\left(0, T ; H_{0}^{1}(\Omega)\right)}+\left\|\partial_{t} v\right\|_{L^{2}\left(0, T ; L^{2}(\Omega)\right)} .
$$

Under assumptions (2.3)-(2.5), the weak form of (2.1) has a unique solution $u_{\varepsilon} \in$ $E$. In fact, the solution is even more regular as $u_{\varepsilon} \in C\left([0, T] ; H_{0}^{1}(\Omega)\right), \partial_{t} u_{\varepsilon} \in$ $C\left([0, T] ; L^{2}(\Omega)\right)([28$, Chap. 3]), if redefined on a set of measure zero.

The discretization of (2.9) with FE is by now standard. Hence we seek a piecewise polynomial solution $u^{h}(t)$ (of degree $l$ ) to the weak form of (2.1) in a finite dimensional subspace of $E$. Classical FE convergence results for the wave equation [9] then yield the optimal a priori error bounds

$$
\left\|\partial_{t} e^{h}\right\|_{L^{\infty}\left(0, T ; L^{2}(\Omega)\right)}+\left\|e^{h}\right\|_{L^{\infty}\left(0, T ; H^{1}(\Omega)\right)} \leq C\left(\frac{h}{\varepsilon}\right)^{l}
$$

for the energy norm and

$$
\left\|e^{h}\right\|_{L^{\infty}\left(0, T ; L^{2}(\Omega)\right)} \leq C\left(\frac{h}{\varepsilon}\right)^{l+1},
$$

for the $L^{2}$ norm, where $e^{h}=u^{\varepsilon}-u^{h}$ and $C$ is independent of $h$ and $\varepsilon$. Hence $h<\varepsilon$ is needed for convergence and the computational cost thus prohibitive when $\varepsilon<<1$. To overcome this difficulty, a well-known remedy is to look for a suitably averaged equation, the homogenized equation - see below -, and approximate its solution numerically. Unfortunately the coefficients (or homogenized tensor) of this homogenized equation can rarely be computed analytically. In Section 3 we shall show how to approximate numerically the effective homogenized solution without first deriving the homogenized equation, by recovering the homogenized tensor "on the fly" with the help of a micro solver defined on suitable sampling domains.

Homogenization theory. Following [13], we briefly review well-known results from homogenization theory for the wave equation. Consider first the elliptic problem associated to (2.9), that is

$$
-\nabla \cdot\left(a^{\varepsilon} \nabla v_{\varepsilon}\right)=F \in \Omega, v_{\varepsilon}=0 \text { on } \partial \Omega .
$$

From the uniform ellipticity and boundedness of $a^{\varepsilon}$ (2.2), we obtain by the LaxMilgram theorem a family of solutions $\left\{v_{\varepsilon}\right\}$ (uniformly bounded independently of $\varepsilon$ ) in $H_{0}^{1}(\Omega)$. Due to the reflexivity of $H_{0}^{1}(\Omega)$, there exists a subsequence of $\left\{v_{\varepsilon}\right\}$ that weakly converges (in $H_{0}^{1}(\Omega)$ ) to a function $v_{0}$. However, this procedure does not yield a limiting equation for $v_{0}$. To prove that $v_{0}$ is the solution of some limiting equation, one usually relies on the notion of $G$ or $H$ convergence (see [33][22],[29]), the former being restricted to symmetric tensors.

A sequence of matrices $\left\{a^{\varepsilon}\right\}$ is said to $G$-converge to a matrix $a^{0}$ (which also satisfies (2.2)) if for any $\psi \in H^{-1}(\Omega)$ the sequence of solution $\left\{v_{\varepsilon}\right\}$ of the above elliptic problem converges weakly in $H_{0}^{1}(\Omega)$ to the solution $v_{0}$ of

$$
-\nabla \cdot\left(a^{0} \nabla v_{0}\right)=\psi \in \Omega, v_{0}=0 \text { on } \partial \Omega .
$$

This convergence is denoted $a^{\varepsilon} \stackrel{G}{\rightarrow} a^{0}$. The importance of $G$-convergence lies in the following compactness result [33][22],[29]: let $\left\{a^{\varepsilon}\right\}$ be a sequence of matrices satisfying 
(2.2). Then there exists a matrix $a^{0}$ satisfying (2.2) and a subsequence $\left\{a^{\varepsilon^{\prime}}\right\}$ which $G$-converges to $a^{0}$.

Consider now the wave equation (2.1) and assume that $a^{\varepsilon}$ satisfies (2.2) and that $a^{\varepsilon} \stackrel{G}{\rightarrow} a^{0}$. Then, the following convergence result holds: $u_{\varepsilon} \rightarrow u_{0}$ weakly* in $L^{\infty}\left(0, T ; H_{0}^{1}(\Omega)\right), \partial_{t} u_{\varepsilon} \rightarrow \partial_{t} u_{0}$ weakly* in $L^{\infty}\left(0, T ; L^{2}(\Omega)\right)$, where $u_{0}$ is the solution of the homogenized problem (2.9)

$$
\begin{aligned}
& \left.\partial_{t t} u_{0}-\nabla \cdot\left(a^{0} \nabla u_{0}\right)=F \text { in } \Omega \times\right] 0, T[ \\
& \left.u_{0}=0 \text { on }\right] 0, T[\times \partial \Omega, \\
& u_{0}(x, 0)=f(x), \quad \partial_{t} u_{0}(x, 0)=g(x), \quad x \in \Omega .
\end{aligned}
$$

Let us end this short review by noting that if $a^{\varepsilon}(x)$ has more structure (e.g. if $a^{\varepsilon}(x)=a(x, x / \varepsilon)=a(x, y)$ is $Y$-periodic in $\left.y\right)$ and if we assume additional regularity (e.g. $a_{i j}(x, y) \in C\left(\bar{\Omega} ; W_{\text {per }}^{1, \infty}(Y)\right)$ for all $\left.i, j=1, \ldots, d\right)$, then the entire sequence $\left\{u_{\varepsilon}\right\}$ weakly converges in the sense described above [26]. In the case of periodic heterogeneities, explicit formulas for the homogenized tensor $a_{0}$ exist, which involve the solution of a boundary value problem, the so-called "cell-problem".

3. Multiscale FEM for the wave equation. In this section, we propose a multiscale FE method for the wave equation, based on the finite element heterogeneous multiscale method (FE-HMM), introduced and analyzed for elliptic and parabolic problems in $[19,1,20]$ - see $[6,7]$ for a review.

3.1. FE space discretization. Macro finite element space. Let $\mathcal{T}_{H}$ be a (macro) partition of $\Omega$ in simplicial or quadrilateral elements $K$ of diameter $H_{K}$, with $H=\max _{K \in \mathcal{T}_{H}} H_{K}$; here, for simplicity, we assume that $\Omega$ is a convex polygon. By macro partition we mean that $H>{ }$ is allowed. For this partition we define a macro FE space

$$
S_{0}^{l}\left(\Omega, \mathcal{T}_{H}\right)=\left\{v^{H} \in H_{0}^{1}(\Omega) ;\left.v^{H}\right|_{K} \in \mathcal{R}^{l}(K), \forall K \in \mathcal{T}_{H}\right\},
$$

where $\mathcal{R}^{l}(K)$ is the space $\mathcal{P}^{l}(K)$ of polynomials on $K$ of total degree at most $l$ if $K$ is a simplex, or the space $\mathcal{Q}^{l}(K)$ of polynomials on $K$ of degree at most $l$ in each variable if $K$ a rectangle. Inside each macro element $K \in \mathcal{T}_{H}$, we consider for $j=1, \ldots, J$ :

- integration points $x_{j, K} \in K$,

- sampling domains $K_{\delta_{j}}=x_{j, K}+\delta I$, where $I=(-1 / 2,1 / 2)^{d}$ and $\delta \geq \varepsilon$,

- quadrature weights $\omega_{j, K}$.

Quadrature formula Let $\hat{K}$ be the reference element and consider for any element of the triangulation $\mathcal{T}_{H}$ the mapping $F_{K}$ (a $C^{1}$-diffeomorphism) such that $K=F_{K}(\hat{K})$. For each $K$, we suppose that the above quadrature weights and integration points are given by

$$
x_{j, K}=F_{K}\left(\hat{x}_{j}\right), \omega_{j}=\hat{\omega}_{j, K} \operatorname{det}\left(\nabla F_{K}\right), \quad j=1, \ldots, J .
$$

Here $\left\{\hat{x}_{j}, \hat{\omega}_{j}\right\}$ is a quadrature formula on $\hat{K}$, which we assume to satisfy:

(Q1) $\hat{\omega}_{j}>0, j=1, \ldots, J, \quad \sum_{j \in J} \hat{\omega}_{j}\left|\nabla \hat{p}\left(\hat{x}_{j}\right)\right|^{2} \geq \hat{\lambda}\|\nabla \hat{p}\|_{L^{2}(\hat{K})}^{2} ;$

(Q2) $\int_{\hat{K}} \hat{q}(x) d x=\sum_{j \in J} \hat{\omega}_{j} \hat{q}\left(\hat{x}_{j}\right), \forall \hat{q}(\hat{x}) \in \mathcal{R}^{\sigma}(\hat{K})$, where $\sigma=\max (2 l-2, l)$ if $\hat{K}$ is a simplicial FE, or $\sigma=\max (2 l-1, l+1)$ if $\hat{K}$ is a rectangular FE.

The standard assumptions (Q1) and (Q2) about the quadrature formula ensure that the optimal convergence rates of the FEM for elliptic problems hold, when using 
numerical integration [15, Chap. 4.1].

For $l=1$ and simplicial finite elements, for instance, we can choose $J=1$ and $\omega_{j}=|K|$.

Micro finite element space. We consider a (micro) partition $\mathcal{T}_{h}$ of each sampling domain $K_{\delta_{j}}$ in simplicial or quadrilateral elements $Q$ of diameter $h_{Q}$ and let $h=$ $\max _{i \in \mathcal{T}_{h}} h_{Q}$. For this partition we define a micro FE space

$$
S^{q}\left(K_{\delta}, \mathcal{T}_{h}\right)=\left\{z_{h} \in W\left(K_{\delta_{j}}\right) ;\left.z_{h}\right|_{T}, \in \mathcal{R}^{r}(Q), Q \in \mathcal{T}_{h}\right\},
$$

where $W\left(K_{\delta_{j}}\right)$ is a Sobolev space whose choice sets the boundary conditions for the micro problems and thus determines the type of coupling between micro and macro problems. For a periodic coupling

$$
W\left(K_{\delta_{j}}\right)=W_{p e r}^{1}\left(K_{\delta_{j}}\right)=\left\{v \in H_{p e r}^{1}\left(K_{\delta_{j}}\right) ; \int_{K_{\delta_{j}}} v d x=0\right\},
$$

and for a coupling through Dirichlet boundary conditions

$$
W\left(K_{\delta_{j}}\right)=H_{0}^{1}\left(K_{\delta_{j}}\right) .
$$

Typically the sampling domains $K_{\delta_{j}}$ are of size $\varepsilon$, that is $\left|K_{\delta_{j}}\right|=O\left(\varepsilon^{d}\right)$, and hence $h<\varepsilon \leq \delta$ holds for the micro mesh size.

Multiscale FE Method. Find $u^{H} \in[0, T] \times S_{0}^{l}\left(\Omega, \mathcal{T}_{H}\right) \rightarrow \mathbb{R}$ such that

$$
\begin{aligned}
& \left(\partial_{t t} u^{H}, v^{H}\right)+B_{H}\left(u^{H}, v^{H}\right)=\left(F(t), v^{H}\right) \quad \forall v^{H} \in S_{0}^{l}\left(\Omega, \mathcal{T}_{H}\right) \\
& \left.u^{H}=0 \text { on } \partial \Omega \times\right] 0, T[ \\
& u^{H}(x, 0)=\Pi_{H}(f(x)), \quad \partial_{t} u^{H}(x, 0)=\Pi_{H}(g(x)), \quad x \in \Omega
\end{aligned}
$$

where

$$
B_{H}\left(u^{H}, v^{H}\right)=\sum_{K \in \mathcal{T}_{H}} \sum_{j=1}^{J} \frac{\omega_{j, K}}{\left|K_{\delta_{j}}\right|} \int_{K_{\delta_{j}}} a^{\varepsilon}(x) \nabla u_{K_{j}}^{h} \cdot \nabla v_{K_{j}}^{h} d x
$$

Here $\Pi_{H}$ denotes the $L^{2}$ - projection on $S_{0}^{l}\left(\Omega, \mathcal{T}_{H}\right)$ and $u_{K_{j}}^{h}, v_{K_{j}}^{h}$ are micro functions defined on the sampling domains $K_{\delta_{j}}$, through (3.7). Note that the factor $1 /\left|K_{\delta_{j}}\right|$ gives the appropriate weight for the contribution of the integrals defined on $K_{\delta}$ instead of $K$.

Micro solver. For every macro element $K$, we determine the additive contribution to the macro stiffness matrix by computing the solutions to the micro-problems, $u_{K_{j}}^{h}$ (respectively $v_{K_{j}}^{h}$ ), on each sampling domain $K_{\delta_{j}}$ : find $u_{K_{j}}^{h}$ such that $\left(u_{K_{j}}^{h}-u_{l i n, K_{j}}^{H}\right) \in$ $S_{h}^{q}\left(K_{\delta_{j}}, \mathcal{T}_{h}\right)$ and

$$
\int_{K_{\delta_{j}}} a^{\varepsilon}(x) \nabla u_{K_{j}}^{h} \cdot \nabla z^{h} d x=0, \quad \forall z^{h} \in S_{h}^{q}\left(K_{\delta_{j}}, \mathcal{I}_{h}\right),
$$

where $S_{h}^{q}\left(K_{\delta_{j}}, \mathcal{T}_{h}\right)$ is the micro FE space defined above and

$$
u_{l i n, K_{j}}^{H}=u^{H}\left(x_{j, K}\right)+\left(x-x_{j, K}\right) \cdot \nabla u^{H}\left(x_{j, K}\right)
$$


is a piecewise linear approximation of $u^{H} \in S_{0}^{l}\left(\Omega, \mathcal{T}_{H}\right)$ about the integration point $x_{j, K}$ (see [20],[6] for details).

REMARK 3.1. The micro stiffness matrices for the numerical solution of (3.7) need only be computed once for each sampling domain, while numerical quadrature is usually needed [15, Chap. 4.1]. A detailed description of the practical implementation of the above numerical method for elliptic and parabolic problems is discussed in [4]. Its extension to the wave equation is straightforward.

Coercivity of the FE-HMM bilinear form. We recall here two results from the analysis of the FE-HMM for elliptic problems (see [1],[20],[6, Sect. 3.3.1] for a proof).

Lemma 3.2. Let $v^{H} \in S_{0}^{l}\left(\Omega, \mathcal{T}_{H}\right)$ and $v^{h}$ be the solutions (3.7) with $S\left(K_{\delta}\right)$ given by (3.3) or (3.4), respectively. Furthermore consider the piecewise linear approximation $v_{\text {lin }}^{H}$ of $v^{H}$ as defined in (3.8). Then we have

$$
\left\|\nabla v_{\text {lin }}^{H}\right\|_{L^{2}\left(K_{\delta}\right)} \leq\left\|\nabla v^{h}\right\|_{L^{2}\left(K_{\delta}\right)} \leq \sqrt{\frac{\Lambda}{\lambda}}\left\|\nabla v_{\text {lin }}^{H}\right\|_{L^{2}\left(K_{\delta}\right)},
$$

where $\lambda, \Lambda$ are defined in (2.2).

The above result implies the following lemma.

Lemma 3.3. Assume that (2.2) and (Q1) hold. Then, the bilinear form $B_{H}(\cdot, \cdot)$ defined in (3.5) is elliptic and bounded, and

$$
\gamma\left\|v^{H}\right\|_{H^{1}(\Omega)}^{2} \leq B_{H}\left(v^{H}, v^{H}\right), \quad\left|B_{H}\left(v^{H}, w_{H}\right)\right| \leq \Gamma\left\|v^{H}\right\|_{H^{1}(\Omega)}\left\|w^{H}\right\|_{H^{1}(\Omega)},
$$

for all $v^{H}, w^{H} \in S_{0}^{l}\left(\Omega, \mathcal{T}_{H}\right)$.

We emphasize that these two Lemmas require no structure assumption (as e.g. periodicity) about the small scale dependence of the tensor $a^{\varepsilon}$.

The semi-discrete Multiscale Method. In the analysis below we shall make use of the so-called semi-discrete multiscale method defined as follows: find $\bar{u}^{H} \in$ $[0, T] \times S_{0}^{l}\left(\Omega, \mathcal{T}_{H}\right) \rightarrow \mathbb{R}$ such that

$$
\begin{aligned}
& \left(\partial_{t t} \bar{u}^{H}, v^{H}\right)+\bar{B}_{H}\left(\bar{u}^{H}, v^{H}\right)=\left(F(t), v^{H}\right) \quad \forall v^{H} \in S_{0}^{l}\left(\Omega, \mathcal{T}_{H}\right) \\
& \left.\bar{u}^{H}=0 \text { on } \partial \Omega \times\right] 0, T[ \\
& \bar{u}^{H}(x, 0)=\Pi_{H}(f(x)), \quad \partial_{t} \bar{u}^{H}(x, 0)=\Pi_{H}(g(x)), \quad x \in \Omega .
\end{aligned}
$$

This problem is similar to (3.11) except for the bilinear form $\bar{B}_{H}(\cdot, \cdot)$ defined for every $v^{H}, w^{H} \in S_{0}^{l}\left(\Omega, \mathcal{T}_{H}\right)$ as

$$
\bar{B}_{H}\left(v^{H}, w^{H}\right)=\sum_{K \in \mathcal{T}_{H}} \sum_{j=1}^{J} \frac{\omega_{j, K}}{\left|K_{\delta_{j}}\right|} \int_{K_{\delta_{j}}} a^{\varepsilon}(x) \nabla v \cdot \nabla w d x,
$$

where the micro functions $v, w$ are not obtained through $\mathrm{FE}$ discretization but instead are the solutions of the continuous counterpart to (3.7): find $v$ (respectively $w$ ) such that $\left(v-v_{l i n, K_{j}}^{H}\right) \in W\left(K_{\delta_{j}}\right)$ and

$$
\int_{K_{\delta_{j}}} a^{\varepsilon}(x) \nabla v \cdot \nabla z d x=0, \quad \forall z \in W\left(K_{\delta_{j}}\right)
$$

with $W\left(K_{\delta_{j}}\right)$ as in (3.3) or (3.4), and $v_{\text {lin }, K_{j}}^{H}$ defined similarly as in (3.8). As for $B_{H}$ in (3.6), one can also prove for $\bar{B}_{H}(\cdot, \cdot)$ that $(3.9)$ with $S_{h}^{q}\left(K_{\delta_{j}}, \mathcal{T}_{h}\right)$ replaced by $W\left(K_{\delta_{j}}\right)$ in (3.7) holds, and thereby infer its ellipticity and boundedness. Clearly, $\bar{u}^{H}$ cannot be obtained in general by a numerical method, as it involves micro functions belonging to an infinite dimensional space, which are not explicitly known. 
4. Convergence Analysis. In this section we analyze the FE-HMM method for the wave equation. We first present our main results, the a-priori error estimates of our numerical method, before proceeding with the detailed analysis.

4.1. Main results. Following [6] we decompose the error $\left\|u_{0}-u^{H}\right\|$ as

$$
\left\|u_{0}-u^{H}\right\| \leq \underbrace{\left\|u_{0}-u_{0}^{H}\right\|}_{\text {err mac }}+\underbrace{\left\|u_{0}^{H}-\bar{u}^{H}\right\|}_{\text {err } \text { mod }}+\underbrace{\left\|\bar{u}^{H}-u^{H}\right\|}_{\text {err mic }},
$$

where $\|\cdot\|$ denotes either the energy or the $L^{2}$ norm. The first term on the right-hand side of (4.1) represents the so-called macro error and involves the solution $u_{0}^{H}(t) \in$ $S_{0}^{l}\left(\Omega, \mathcal{T}_{H}\right)$ of (4.2) (standard FEM with numerical integration for the homogenized wave equation). The second term corresponds to the so-called modeling error and involves the solution $\bar{u}^{H}(t) \in S_{0}^{l}\left(\Omega, \mathcal{T}_{H}\right)$ of (3.11). Finally, the last term represents the so-called micro error and involves the solution $u^{H}(t) \in S_{0}^{l}\left(\Omega, \mathcal{T}_{H}\right)$ of $(3.5)$, that is the numerical solution of our FE-HMM method.

We postpone the analysis of the modeling and the micro errors. For the macro error, we can use standard error analysis techniques for FEM with numerical integration - see [15] for the elliptic case, [31] for the parabolic case, and [10] for the hyperbolic case. Consider the standard FEM with numerical integration for the homogenized wave equation: find $u_{0}^{H} \in[0, T] \times S_{0}^{1}\left(\Omega, \mathcal{T}_{H}\right) \rightarrow \mathbb{R}$ such that

$$
\begin{aligned}
& \left(\partial_{t t} u_{0}^{H}, v^{H}\right)+B_{0, H}\left(u_{0}^{H}, v^{H}\right)=\left(F(t), v^{H}\right) \quad \forall v^{H} \in S_{0}^{1}\left(\Omega, \mathcal{T}_{H}\right) \\
& \left.u_{0}^{H}=0 \text { on } \partial \Omega \times\right] 0, T[ \\
& u_{0}^{H}(x, 0)=\Pi_{H}(f(x)), \quad \partial_{t} u_{0}^{H}(x, 0)=\Pi_{H}(g(x)), \quad x \in \Omega,
\end{aligned}
$$

where $B_{0, H}(\cdot, \cdot)$ is defined for every $v^{H}, w^{H} \in S_{0}^{l}\left(\Omega, \mathcal{T}_{H}\right)$ by

$$
B_{0, H}\left(v^{H}, w^{H}\right)=\sum_{K \in \mathcal{T}_{H}} \sum_{j=1}^{J} \omega_{j, K} a^{0}\left(x_{j, K}\right) \nabla v^{H}\left(x_{j, K}\right) \nabla w^{H}\left(x_{j, K}\right) .
$$

Optimal error estimates for the macroscopic error $e_{0}=u_{0}-u_{0}^{H}$, where $u_{0}$ is the solution of (2.9), were proved by Baker and Dougalis [10]. They are repeated here for the sake of completeness.

Proposition 4.1. Let $u_{0}^{H}$ be be the solution of (4.2) and $u_{0}$ be the solution of (2.9). Assume that the quadrature formula satisfies (Q1) and (Q2), the homogenized tensor satisfies $a_{i j}^{0} \in W^{l+1, \infty}(\Omega), i, j=1, \ldots, d$, and

$$
\partial_{t^{k}} F \in L^{2}\left(0, T ; W^{l+1, q}(\Omega)\right), k=0,1,2 .
$$

If the solution $u_{0}$ of (2.9) satisfies

$$
\partial_{t^{k}} u_{0} \in L^{2}\left(0, T ; W^{l+1, q}(\Omega)\right), 0 \leq k \leq 3,
$$

then the error $e_{0}=u_{0}-u_{0}^{H}$ satisfies the estimate

$$
\left\|e_{0}\right\|_{L^{\infty}\left(0, T ; L^{2}(\Omega)\right)} \leq C H^{l+1}\left(\max _{0 \leq k \leq 3}\left\|\partial_{t^{k}} u_{0}\right\|_{L^{2}\left(0, T ; W^{q, l+1}(\Omega)\right)}\right) .
$$

If the solution $u_{0}$ of (2.9) satisfies

$$
\partial_{t^{k}} u_{0} \in L^{2}\left(0, T ; W^{l+1, q}(\Omega)\right), 0 \leq k \leq 4,
$$


then the error $e_{0}=u_{0}-u_{0}^{H}$ satisfies the estimate

$$
\left\|\partial_{t} e_{0}\right\|_{L^{\infty}\left(0, T ; L^{2}(\Omega)\right)}+\left\|e_{0}\right\|_{L^{\infty}\left(0, T ; H^{1}(\Omega)\right)} \leq C H^{l}\left(\max _{0 \leq k \leq 4}\left\|\partial_{t^{k}} u_{0}\right\|_{L^{2}\left(0, T ; W^{l+1, q}(\Omega)\right)}\right) .
$$

REMARK 4.2. On the right of (4.7), a term of the form

$$
\left\|f-\Pi_{H} f\right\|_{H^{1}(\Omega)}+\left\|\partial_{t} g-\partial_{t} \Pi_{H} g\right\|_{L^{2}(\Omega)}
$$

usually appears, while a term of the form

$$
\left\|f-\Pi_{H} f\right\|_{L^{2}(\Omega)}
$$

usually appears on the right of (4.5). Since our assumptions on $u, \partial_{t} u$ imply that $u, \partial_{t} u \in C\left([0, T] ; W^{l+1, q}(\Omega)\right)$ (see [21, Sect. 5.9.2]), standard approximation results imply that (4.8) is bounded by $C H^{l}$ and that (4.9) is bounded by $C H^{l+1}$.

We now state our main result.

THEOREM 4.3. Let $u^{H}$ be be the solution of (3.5), $u_{0}$ the solution of (2.9) and the error $e^{H}=u_{0}-u^{H}$ and suppose that the assumptions of Proposition 4.1 hold. Then we have

$$
\begin{aligned}
& \left\|\partial_{t} e^{H}\right\|_{L^{\infty}\left(0, T ; L^{2}(\Omega)\right)}+\left\|e^{H}\right\|_{L^{\infty}\left(0, T ; H^{1}(\Omega)\right)} \leq C_{1} H^{l}\left(\max _{0 \leq k \leq 4}\left\|\partial_{t^{k}} u_{0}\right\|_{L^{2}\left(0, T ; W^{q, l+1}(\Omega)\right)}\right) \\
& +C_{2} \sup _{K \in \mathcal{T}_{H}, x_{j, K} \in K}\left\|a^{0}\left(x_{j, K}\right)-a_{K}^{0}\left(x_{j, K}\right)\right\|_{F} .
\end{aligned}
$$

For the $L^{2}$ error, we have the estimate

$$
\begin{aligned}
& \left\|e^{H}\right\|_{L^{\infty}\left(0, T ; L^{2}(\Omega)\right)} \leq C_{1} H^{l+1}\left(\max _{0 \leq k \leq 3}\left\|\partial_{t^{k}} u_{0}\right\|_{L^{2}\left(0, T ; W^{q, l+1}(\Omega)\right)}\right)+ \\
& C_{2} \sup _{K \in \mathcal{T}_{H}, x_{j, K} \in K}\left\|a^{0}\left(x_{j, K}\right)-a_{K}^{0}\left(x_{j, K}\right)\right\|_{F} .
\end{aligned}
$$

Here $\|\cdot\|_{F}$ denotes the Frobenius norm and the constants $C_{1}$ and $C_{2}$ depend on $\lambda, \Lambda, T, F, f, g$ but not on $H, h$, or $\varepsilon$. Theorem 4.3 implies optimal convergence rates in the $L^{2}$ and $H^{1}$ norms with respect to the macro meshsize $H$. In both estimates the second term on the right side does not appear in standard FEM and stems from the modeling and micro errors mentioned in (4.1). To clarify that connection, we further decompose it as

$$
\left\|a^{0}\left(x_{j, K}\right)-a_{K}^{0}\left(x_{j, K}\right)\right\|_{F} \leq \underbrace{\left\|a^{0}\left(x_{j, K}\right)-\bar{a}_{K}^{0}\left(x_{j, K}\right)\right\|_{F}}_{\text {err } \text { mod }}+\underbrace{\left\|\bar{a}^{0}\left(x_{j, K}\right)-a_{K}^{0}\left(x_{j, K}\right)\right\|_{F}}_{\text {err } r_{\text {mic }}} .
$$

The tensor $a^{0}\left(x_{j, K}\right)$ is the homogenized tensor in (2.9) evaluated at the quadrature point $x_{j, K}$. The tensors $\bar{a}^{0}\left(x_{j, K}\right)$ and $a_{K}^{0}\left(x_{j, K}\right)$ (numerical approximations of $\left.a^{0}\left(x_{j, K}\right)\right)$ are defined in (4.15) and (4.16), respectively, and the above error estimate is discussed in Section 4.3. The first term of the right-hand side of the above inequality $\left(e r r_{m o d}\right)$ is the so-called modeling error and describes how well the upscaling procedure (multiscale method) captures the effective coefficients of (2.9). The second term $\left(e r r_{m i c}\right)$ describes the error due to the micro FEM. This term can be analyzed without further assumptions on the spatial structure (e.g. periodicity, random stationarity), but some regularity assumptions about the oscillating tensor $a^{\varepsilon}$ are needed (see Remark 4.7). 
If the solutions of the micro cell problems (3.7) are sufficiently regular - see Remark 4.7 and Lemma 4.8 below for a precise statement - err mic can be quantified and Theorem 4.3 implies the optimal error bounds

$$
\begin{aligned}
& \left\|\partial_{t} e^{H}\right\|_{L^{\infty}\left(0, T ; L^{2}(\Omega)\right)}+\left\|e^{H}\right\|_{L^{\infty}\left(0, T ; H^{1}(\Omega)\right)} \leq C\left(H^{l}+\left(\frac{h}{\varepsilon}\right)^{2 q}+e r r_{m o d}\right), \\
& \left\|e^{H}\right\|_{L^{\infty}\left(0, T ; L^{2}(\Omega)\right)} \leq C\left(H^{l+1}+\left(\frac{h}{\varepsilon}\right)^{2 q}+e r r_{\text {mod }}\right)
\end{aligned}
$$

where $C$ is independent of $H, h, \varepsilon$. In the periodic or random stationary case $e r r_{\text {mod }}$ can also be quantified (see Lemmas 4.10 and 4.11).

4.2. Preliminaries. First, we define the tensors $a^{0}\left(x_{j, K}\right), \bar{a}^{0}\left(x_{j, K}\right), a_{K}^{0}\left(x_{j, K}\right)$ mentioned previously. Following [5],[6] we consider for each vector $\mathbf{e}_{\mathbf{i}}, i=1, \ldots, d$ of the canonical basis $\mathbb{R}^{d}$ the function $\psi_{K_{\delta_{j}}}^{i}$, solution of the micro problem similar to (3.7) with modified right-hand side

$$
\int_{K_{\delta_{j}}} a^{\varepsilon}(x) \nabla \psi_{K_{\delta_{j}}}^{i} \nabla z d x=-\int_{K_{\delta_{j}}} a^{\varepsilon}(x) \mathbf{e}_{\mathbf{i}} \cdot \nabla z d x, \forall z \in W\left(K_{\delta_{j}}\right),
$$

where $W\left(K_{\delta_{j}}\right)$ is defined in (3.3) or (3.4). Similarly we consider (4.14) in the FE space $S^{q}\left(K_{\delta}, \mathcal{T}_{h}\right)$ and denote its corresponding solution by $\psi_{K_{\delta_{j}}}^{i, h}$. At each quadrature point $x_{j, K} \in K$, we define a so-called numerically homogenized tensor as

$$
a_{K}^{0}\left(x_{j, K}\right)=\frac{1}{\left|K_{\delta_{j}}\right|} \int_{K_{\delta_{j}}} a^{\varepsilon}(x)\left(I+J_{\psi_{K_{\delta_{j}}}^{h}}^{T}(x)\right) d x
$$

where $J_{\psi_{\delta_{\delta_{j}}}^{h}(x)}$ is a $d \times d$ matrix with entries $\left(J_{\psi_{K_{\delta_{j}}}^{h}(x)}\right)_{i \ell}=\left(\partial \psi_{K_{\delta_{j}}}^{i, h}\right) /\left(\partial x_{\ell}\right)$. We also define

$$
\bar{a}_{K}^{0}\left(x_{j, K}\right)=\frac{1}{\left|K_{\delta_{j}}\right|} \int_{K_{\delta_{j}}} a^{\varepsilon}(x)\left(I+J_{\psi_{K_{\delta_{j}}}}^{T}(x)\right) d x,
$$

where $J_{\psi_{K_{\delta_{j}}}(x)}$ is defined similarly as $J_{\psi_{K_{\delta_{j}}}^{h}(x)}$, but with $\psi_{K_{\delta_{j}}}^{i, h}$ replaced by $\psi_{K_{\delta_{j}}}^{i}$.

By using the numerically homogenized tensor (4.15) and the results of [5] (see also Lemmas 11 and 12 of [7]) we obtain the following useful reformulation of the bilinear form $B_{H}(\cdot, \cdot)$ in $(3.5)$.

Lemma 4.4. The bilinear form $B_{H}(\cdot, \cdot)$ defined in (3.6) can be rewritten as

$$
B_{H}\left(v^{H}, w^{H}\right)=\sum_{K \in \mathcal{T}_{H}} \sum_{j=1}^{J} \omega_{j, K} a_{K}^{0}\left(x_{j, K}\right) \nabla v^{H}\left(x_{j, K}\right) \nabla w^{H}\left(x_{j, K}\right) .
$$

Likewise, we have for the semi-discrete bilinear form a similar result,

Lemma 4.5. The bilinear form $\bar{B}_{H}(\cdot, \cdot)$ defined in $(3.12)$ can be rewritten as

$$
\bar{B}_{H}\left(v^{H}, w^{H}\right)=\sum_{K \in \mathcal{T}_{H}} \sum_{j=1}^{J} \omega_{j, K} \bar{a}_{K}^{0}\left(x_{j, K}\right) \nabla v^{H}\left(x_{j, K}\right) \nabla w^{H}\left(x_{j, K}\right) .
$$


4.3. A priori error analysis for the FE-HMM. In this section we prove our main theorem. Recall that the analysis of FE-HMM methods for elliptic problems use results from non-conforming FEM; in particular, Strang-type lemmas play an important role [1],[20],[6],[7]. Thus we shall first derive a similar result for the wave equation which, to the best of our knowledge, is not available in the literature. Its proof is given in the appendix.

Lemma 4.6. Let $V \subset H_{0}^{1}(\Omega), \hat{f}, \hat{g} \in V, F, \partial_{t} F \in L^{2}\left(0, T ; L^{2}(\Omega)\right), T>0$, and $u_{1}$, $u_{2}$ be the (unique) solutions of

$$
\begin{aligned}
& \left.\left(\partial_{t t} u_{1}, v\right)+B_{1}\left(u_{1}, v\right)=(F, v), \quad \forall v \in V, \quad t \in\right] 0, T[ \\
& \left.u_{1}=0 \quad \text { on } \partial \Omega \times\right] 0, T[ \\
& u_{1}(x, 0)=\hat{f}(x), \quad \partial_{t} u_{1}(x, 0)=\hat{g}(x), \quad x \in \Omega,
\end{aligned}
$$

and

$$
\begin{aligned}
& \left.\left(\partial_{t t} u_{2}, v\right)+B_{2}\left(u_{2}, v\right)=(F, v), \quad \forall v \in V, \quad t \in\right] 0, T[ \\
& \left.u_{2}=0 \quad \text { on } \partial \Omega \times\right] 0, T[ \\
& u_{2}(x, 0)=\hat{f}(x), \quad \partial_{t} u_{2}(x, 0)=\hat{g}(x), \quad x \in \Omega,
\end{aligned}
$$

respectively, where the two bilinear forms $B_{1}(\cdot, \cdot), B_{2}(\cdot, \cdot)$ satisfy

$$
\gamma_{1}\|v\|_{H^{1}}^{2} \leq B_{i}(v, v), \quad\left|B_{i}(v, w)\right| \leq \gamma_{2}\|v\|_{H^{1}}\|w\|_{H^{1}}, \quad i=1,2 .
$$

Assume that

$$
\partial_{t}^{k} u_{j} \in L^{2}\left(0, T ; H^{1}(\Omega)\right), \quad 0 \leq k \leq 2, \quad j=1,2,
$$

and that

$$
\left|B_{1}(v, w)-B_{2}(v, w)\right| \leq \eta\|v\|_{H^{1}(\Omega)}\|w\|_{H^{1}(\Omega)} \quad \forall v, w \in V .
$$

Then,

$$
\left\|\partial_{t}\left(u_{1}-u_{2}\right)\right\|_{L^{\infty}\left(0, T ; L^{2}(\Omega)\right)}+\left\|u_{1}-u_{2}\right\|_{L^{\infty}\left(0, T ; H^{1}(\Omega)\right)} \leq C \eta,
$$

where $C=C\left(\gamma_{1}, \gamma_{2}, T, \hat{f}, \hat{g}\right)$ is independent of $\eta$. We now prove our main result.

Proof of Theorem 4.3 By using the continuity of $B_{0, H}(\cdot, \cdot)$ defined in (3.11) and $B_{H}(\cdot, \cdot)$ defined in $(3.6)$, we have

$$
\begin{aligned}
& \left|B_{0, H}\left(v^{H}, w^{H}\right)-B_{H}\left(v^{H}, w^{H}\right)\right| \\
& \leq \sum_{K \in \mathcal{T}_{H}}\left|\sum_{j=1}^{J} \omega_{j, K}\left(a^{0}\left(x_{j, K}\right)-a_{K}^{0}\left(x_{j, K}\right)\right) \nabla v^{H}\left(x_{j, K}\right) \nabla w^{H}\left(x_{j}\right)\right| \\
& \leq \sup _{K \in \mathcal{T}_{H}, x_{j, K} \in K}\left\|a^{0}\left(x_{j, K}\right)-a_{K}^{0}\left(x_{j, K}\right)\right\|_{F} \sqrt{\sum_{K \in \mathcal{T}_{H}} \sum_{j=1}^{J} \omega_{j, K}\left|\nabla v^{H}\left(x_{j, K}\right)\right|^{2}} \\
& \cdot \sqrt{\sum_{K \in \mathcal{T}_{H}} \sum_{j=1}^{J} \omega_{j, K}\left|\nabla w^{H}\left(x_{j, K}\right)\right|^{2}} \\
& \leq \sup _{K \in \mathcal{T}_{H}, x_{j, K} \in K}\left\|a^{0}\left(x_{j, K}\right)-a_{K}^{0}\left(x_{j, K}\right)\right\|_{F}\left\|v^{H}\right\|_{H^{1}(\Omega)}\left\|w^{H}\right\|_{H^{1}(\Omega)} .
\end{aligned}
$$


Now let $u_{0}^{H}$ be be the solution of (4.2) and $u^{H}$ the solution of (3.5). From Lemma 4.6 we thus obtain

$$
\begin{aligned}
& \left\|\partial_{t}\left(u_{0}^{H}-u^{H}\right)\right\|_{L^{\infty}\left(0, T ; L^{2}(\Omega)\right)}+\left\|u_{0}^{H}-u^{H}\right\|_{L^{\infty}\left(0, T ; H^{1}(\Omega)\right)} \\
& \leq C \sup _{K \in \mathcal{T}_{H}, x_{j, K} \in K}\left\|a^{0}\left(x_{j, K}\right)-a_{K}^{0}\left(x_{j, K}\right)\right\|_{F},
\end{aligned}
$$

where $C=C(\lambda, \Lambda, T)$. To conclude the proof we use the triangle inequality

$$
\begin{aligned}
& \left\|\partial_{t}\left(u_{0}-u^{H}\right)\right\|_{L^{\infty}\left(0, T ; L^{2}(\Omega)\right)}+\left\|u_{0}-u^{H}\right\|_{L^{\infty}\left(0, T ; H^{1}(\Omega)\right)} \\
& \leq\left\|\partial_{t}\left(u_{0}-u_{0}^{H}\right)\right\|_{L^{\infty}\left(0, T ; L^{2}(\Omega)\right)}+\left\|u_{0}-u_{0}^{H}\right\|_{L^{\infty}\left(0, T ; H^{1}(\Omega)\right)} \\
& +\left\|\partial_{t}\left(u_{0}^{H}-u^{H}\right)\right\|_{L^{\infty}\left(0, T ; L^{2}(\Omega)\right)}+\left\|u_{0}^{H}-u^{H}\right\|_{L^{\infty}\left(0, T ; H^{1}(\Omega)\right)} .
\end{aligned}
$$

The last two terms of the right-hand side of this inequality can be estimated by (4.24). For the first and second term, however, we use Proposition 4.1.

The estimate with respect to the $L^{2}$ norm in (4.11) is obtained again from the triangle inequality, the estimate in (4.5), and the obvious estimate

$$
\left\|u_{0}-u_{0}^{H}\right\|_{L^{\infty}\left(0, T ; L^{2}(\Omega)\right)} \leq C \sup _{K \in \mathcal{T}_{H}, x_{j, K} \in K}\left\|a^{0}\left(x_{j, K}\right)-a_{K}^{0}\left(x_{j, K}\right)\right\|_{F},
$$

which follows from (4.24).

Again, we emphasize that the result of Theorem 4.3 requires no assumption on the spatial structure of the small-scale tensor $a^{\varepsilon}(x)$, such as periodicity or random stationarity. As already mentioned at the end of Section 4.1, Theorem 4.3 involves the unusual term $\left\|a^{0}\left(x_{j, K}\right)-a_{K}^{0}\left(x_{j, K}\right)\right\|_{F}$, which remains to be studied. To do so, we recall the decomposition:

$$
\begin{aligned}
\sup _{K \in \mathcal{T}_{H}, x_{j, K} \in K}\left\|a^{0}\left(x_{j, K}\right)-a_{K}^{0}\left(x_{j, K}\right)\right\|_{F} & \leq \sup _{K \in \mathcal{T}_{H}, x_{j, K} \in K} \underbrace{\left\|a^{0}\left(x_{j, K}\right)-\bar{a}_{K}^{0}\left(x_{j, K}\right)\right\|_{F}}_{e r r_{\text {mod }}\left(x_{j, K}, K\right)} \\
& +\sup _{K \in \mathcal{T}_{H}, x_{j, K} \in K} \underbrace{\left\|\bar{a}^{0}\left(x_{j, K}\right)-a_{K}^{0}\left(x_{j, K}\right)\right\|_{F}}_{\operatorname{err}_{\text {mic }}\left(x_{j, K}, K\right)}
\end{aligned}
$$

Micro error. No assumptions about the spatial structure of $a^{\varepsilon}$ are required to analyze the micro error. Some regularity on the small scales, however, is needed. Hence we shall assume that the solution $\psi_{K_{\delta_{j}}}^{i}$ of the micro problem (4.14) satisfies

$$
\left|\psi_{K_{j}}^{i}\right|_{H^{q+1}\left(K_{\delta_{j}}\right)} \leq C \varepsilon^{-q} \sqrt{\left|K_{\delta_{j}}\right|},
$$

where $C$ is independent of $\varepsilon$, of the quadrature points $x_{j, K}$, and the domain $K_{\delta_{j}}$.

REMARK 4.7. Without any further knowledge about the structure of the oscillating tensor $a^{\varepsilon}$, (4.27) follows for $q=1$ from classical $H^{2}$ regularity results ([27, Chap. 2.6]), provided $\left|a_{i j}^{\varepsilon}\right|_{W^{1, \infty}(\Omega)} \leq C \varepsilon^{-1}$ for $i, j=1, \ldots, d$ and Dirichlet boundary conditions are imposed in (4.14). Note that $\left.a_{i j}^{\varepsilon}\right|_{K} \in W^{1, \infty}(K) \forall K \in \mathcal{T}_{H}$ and $\left|a_{i j}^{\varepsilon}\right|_{W^{1, \infty}(K)} \leq C_{K} \varepsilon^{-1}$ are sufficient, if the macro mesh is aligned with the (possible) discontinuities of $a^{\varepsilon}$ (see [5] for details).

If $a^{\varepsilon}=a(x, x / \varepsilon)=a(x, y)$ is $Y$-periodic in $y$ and $\delta / \varepsilon \in \mathbb{N}$, then (4.27) can be established for any given $q$, provided that $a^{\varepsilon}$ is sufficiently smooth, by following classical regularity results for periodic boundary value problems (see [12, Chap. 3] and also [3]). 
Following [5, Lemma 5.1],[7, Lemma 6], we thus obtain

LEMMA 4.8. Suppose that the solution $\psi_{K_{\delta_{j}}}^{i}$ of the cell problem (4.14) satisfies (4.27). Then,

$$
\sup _{K \in \mathcal{T}_{H}, x_{j, K} \in K}\left\|\bar{a}^{0}\left(x_{j, K}\right)-a_{K}^{0}\left(x_{j, K}\right)\right\|_{F} \leq C\left(\frac{h}{\varepsilon}\right)^{2 q},
$$

where $C$ depends only on the domain $\Omega$, the dimension $d$, the constant $C$ in (4.27) and $\lambda, \Lambda$ in (2.2).

REMARK 4.9. We emphasize that $\hat{h}=h / \varepsilon$ is independent of $\varepsilon$. Indeed, if we use $N_{\text {micro }}$ elements in each space dimension for the discretization of the sampling domains, we have $h=\delta / N_{\text {micro }}$ and therefore $\hat{h}=(\delta / \varepsilon) \cdot\left(1 / N_{\text {micro }}\right)$. Since $\delta$ scales with $\varepsilon$, typically $\delta=C \varepsilon$ (with a constant $C$ of moderate size), we have $\hat{h}=\left(C / N_{\text {micro }}\right)$.

Let $M_{\text {macro }}$ denote the number of degrees of freedom (DOF) of the macro FEM and by $M_{\text {micro }}$ the number of DOF of the micro FEMs. Then the overall complexity is proportional to $\left(M_{\text {macro }}\right)^{\alpha_{1}} \times\left(M_{\text {micro }}\right)^{\alpha_{2}}$, with suitable $\alpha_{1}, \alpha_{2}$ depending on the norm for the error and the type of macro and micro FEs; in general, it is superlinear. In contrast, the memory requirement is only proportional to $M_{\text {macro }}+M_{\text {micro }}$, because the micro problems are independent of one another and thus can be solved one at a time.

Coupling the macro FE method with "fast micro solvers", such as spectral methods, considerably reduces the computational cost of the method even further, provided $a^{\varepsilon}$ is sufficiently regular. This approach was proposed in [3] for elliptic problems and can be extended to the wave equation.

Modeling error. Next, we discuss the so-called modeling error. Here structure assumptions have to be made if we wish quantitative bounds.

Any results concerning the approximation of the homogenized tensor with artificial micro boundary conditions (e.g. Dirichlet) and oversampling could be readily used in our analysis and would permit to estimate the modeling error in more general situations. For simplicity, we consider the situation of a non uniformly periodic tensor of the form

$$
a^{\varepsilon}=a(x, x / \varepsilon)=a(x, y) \quad Y \text {-periodic in } y,
$$

where we set $Y=(0,1)^{d}$. We also assume

$$
a_{i j}(x, y) \in \mathcal{C}\left(\bar{\Omega} ; W_{p e r}^{1, \infty}(Y)\right), \text { for all } i, j=1, \ldots, d .
$$

We shall now distinguish two cases.

If $\delta / \varepsilon \in \mathbb{N}$, we have

LEMmA 4.10. Suppose $\psi_{K_{\delta_{j}}}^{i}$ is the solution of the cell problem (4.14) in $W_{p e r}^{1}\left(K_{\delta_{j}}\right)$ and that both (4.29) and (4.30) hold. Then,

$$
\sup _{K \in \mathcal{T}_{H}, x_{j, K} \in K}\left\|a^{0}\left(x_{j, K}\right)-\bar{a}_{K}^{0}\left(x_{j, K}\right)\right\|_{F} \leq C \varepsilon,
$$

where $C$ is independent of $H, h, \varepsilon$. Moreover if the dependence of $a(x, x / \varepsilon)$ on the slow variable is explicit and one collocates the slow variable of the tensor at quadrature points $a\left(x_{j, K}, x / \varepsilon\right)$ in the FE-HMM macro bilinear form (3.5) and in the micro problems (3.7), then in fact we have

$$
\sup _{K \in \mathcal{T}_{H}, x_{j, K} \in K}\left\|a^{0}\left(x_{j, K}\right)-\bar{a}_{K}^{0}\left(x_{j, K}\right)\right\|_{F}=0 .
$$


Proof. The proof can be obtained following the argument of [6, Proposition 14] and is omitted here.

If $\delta / \varepsilon \notin \mathbb{N}$, we have

LEMMA 4.11. Suppose $\psi_{K_{\delta_{j}}}^{i}$ is the solution of the cell problem (4.14) in the space $H_{0}^{1}\left(K_{\delta_{j}}\right)$ and suppose that both (4.29) and (4.30) hold. Then

$$
\sup _{K \in \mathcal{T}_{H}, x_{j, K} \in K}\left\|a^{0}\left(x_{j, K}\right)-\bar{a}_{K}^{0}\left(x_{j, K}\right)\right\|_{F} \leq C\left(\delta+\frac{\varepsilon}{\delta}\right) .
$$

Proof. The proof can be obtained following the argument of [20, Theorem 3.2].

5. Numerical experiments. We shall now apply our FE-HMM to two simple one-dimensional problems to illustrate its versatility and corroborate the convergence rates proved in Theorem 4.3. First, we consider a model problem with a periodic highly oscillatory medium, where the analytical solution from classical homogenization theory is explicitly known. Second, we consider wave propagation through a highly oscillatory but nonperiodic medium, which appears discontinuous at the macroscale; here, we compare the FE-HMM solution with a reference solution on a highly refined mesh. In both cases we choose the standard second-order leap-frog scheme for the time discretization with constant step size $\Delta t$.
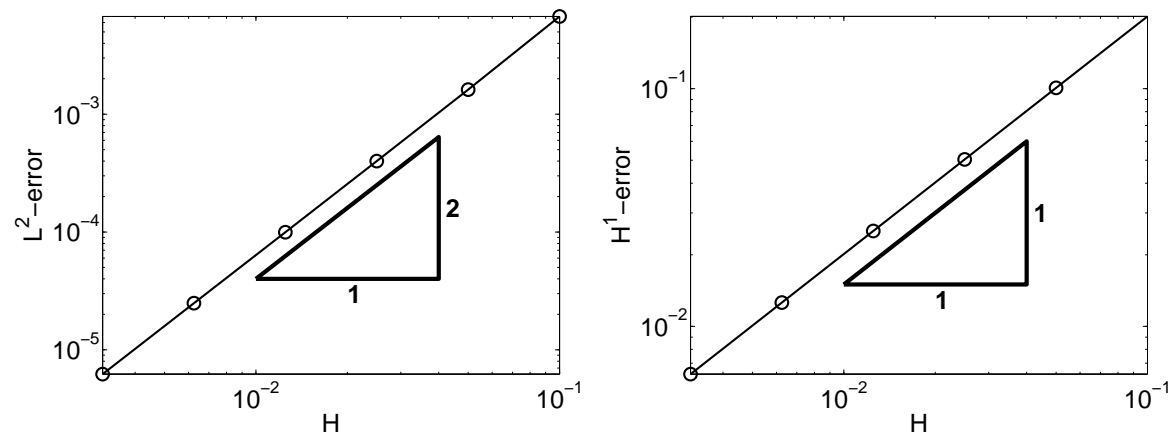

FIG. 5.1. Periodic medium. The error $\left\|u_{0}-u^{H}\right\|$ between the FE-HMM solution $u^{H}$ and the homogenized solution $u^{0}$ is shown vs. the macro mesh size $H$, with simultaneous refinement of $H$ and $h$ : the $L^{2}$-error (left) and the $H^{1}$-error (right).

5.1. Periodic medium. We consider (2.9) in the unit interval $\Omega=] 0,1[$ until the final time $T=2$ with the initial conditions $f(x)=\sin (\pi x)$ and $g(x)=0$. The squared velocity field

$$
a^{\varepsilon}(x)=\frac{\sqrt{17}}{4}+\frac{1}{4} \sin \left(2 \pi \frac{x}{\varepsilon}\right)
$$

is periodic and highly oscillatory for increasingly smaller values of $\varepsilon$. The coefficients are chosen such that the homogenized wave speed $\sqrt{a^{0}}=1$; hence, the homogenized solution is explicitly known here, $u^{0}(x, t)=\sin (\pi x t)$. We now let $\varepsilon=4 \cdot 10^{-4}$ and consider a sequence of equidistant macro partitions $\mathcal{T}_{H}$ of $\Omega$, indexed by $k=1,2, \ldots, 6$, each with mesh size $H=0.2 \cdot 2^{-k}$. Inside each macro interval, a single sampling domain $K_{\delta}$ of diameter $\delta=4 \cdot 10^{-4}$ is centered about the mid-point; it is partitioned into equidistant sub-intervals of size $h=0.2 \delta \cdot 2^{-k}$. Both the macro and the micro 
FE spaces consist of standard continuous piecewise linear functions with $\ell=r=1$. We recall that the convergence rate and the computational complexity of the micro FEMs rely on the scaled mesh size $\hat{h}=h / \varepsilon$ which is independent of $\varepsilon$ (see Remark $(4.9))$.

In Figure 5.1, the $L^{2}$ and the $H^{1}$ errors between the exact homogenized and the FE-HMM solutions are shown for the above sequence of meshes, with simultaneous refinement in $H$ and $h$, and a time step $\Delta t=0.1 \cdot 2^{-k}$. We observe the expected overall convergence of order one in the $H^{1}$ norm and of order two in the $L^{2}$ norm, which corroborates the theoretical bounds from Theorem 4.3.
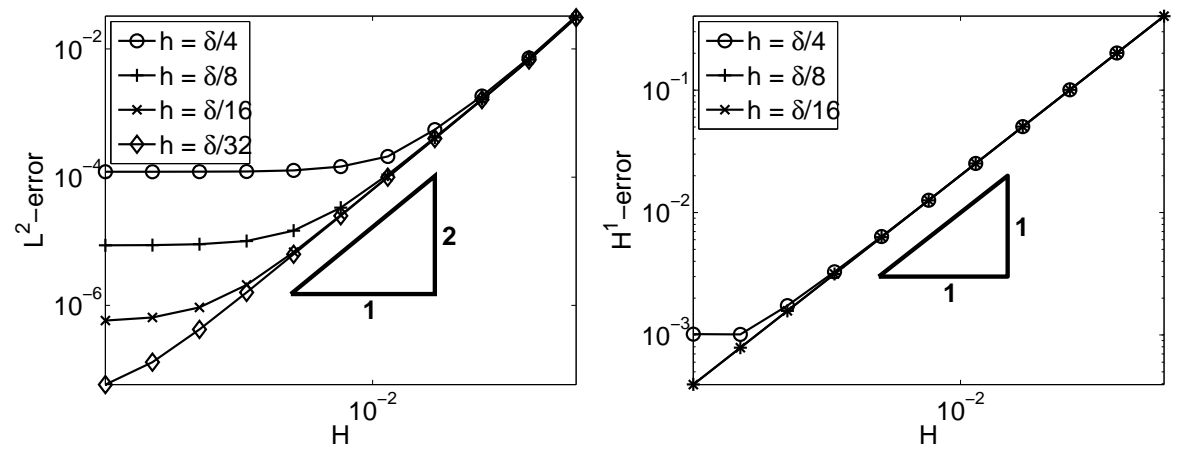

FIG. 5.2. Periodic medium. The error $\left\|u_{0}-u^{H}\right\|$ between the FE-HMM solution $u^{H}$ and the homogenized solution $u^{0}$ is shown vs. the macro mesh size $H$, while the micro mesh size $h$ is kept fixed: the $L^{2}$-error (left) and the $H^{1}$-error (right).

What if only the macro mesh size $H$ is refined while the micro mesh size $h$ is kept fixed? According to the error estimates from Theorem 4.3, we expect the second term on the right side of (4.12) and (4.13), which involves the error due to the micro FEM, to dominate and inhibit convergence as $H$ tends to zero. To illustrate this behavior, we consider four different micro meshes with mesh size $h=\delta \cdot 2^{-(m+1)}, m=1,2,3,4$. Then for each fixed micro mesh, we compute the numerical solutions for the sequence of macro meshes indexed by $k=1,2, \ldots, 11$, each with mesh size $H=0.4 \cdot 2^{-k}$ and with time step $\Delta t=0.2 \cdot 2^{-k}$. Again for $\varepsilon=\delta=4 \cdot 10^{-4}$, the results are shown in Figure 5.2. Although choosing a smaller value for $h$ always improves the accuracy, our results demonstrate that overall convergence to the homogenized solution cannot be achieved without simultaneous refinement of both the macro and the micro FE meshes.

5.2. Heterogeneous medium. Next, we consider $(2.9)$ in $\Omega=]-3,5[$ until the final time $T=3$, where the initial conditions correspond to a right-moving Gaussian pulse,

$$
f(x)=\left\{\begin{array}{ll}
e^{-\frac{x^{2}}{\sigma^{2}}} & x \in[-0.5,0.5] \\
0 & \text { elsewhere }
\end{array},\right.
$$

with $\sigma$ such that $f( \pm 0.5)$ equals machine precision, and

$$
g(x)= \begin{cases}\frac{2 x}{\sigma^{2}} e^{-\frac{x^{2}}{\sigma^{2}}} & x \in[-0.5,0.5] \\ 0 & \text { elsewhere }\end{cases}
$$


The squared velocity field $a^{\varepsilon}(x)$, shown in Figure 5.3, is no longer periodic but nonetheless highly oscillatory for increasingly smaller values of $\varepsilon$. It is given by

$$
a^{\varepsilon}(x)=\left\{\begin{array}{ll}
2+\sqrt{2}+\sin \left(\frac{2 \pi x}{\varepsilon}\right) & \text { for } x>0 \text { and } x \in(n-0.5, n) \text { for a } n \in \mathbb{N} \\
\sqrt{2}+\sin \left(\frac{2 \pi x}{\varepsilon}\right) & \text { elsewhere }
\end{array} .\right.
$$

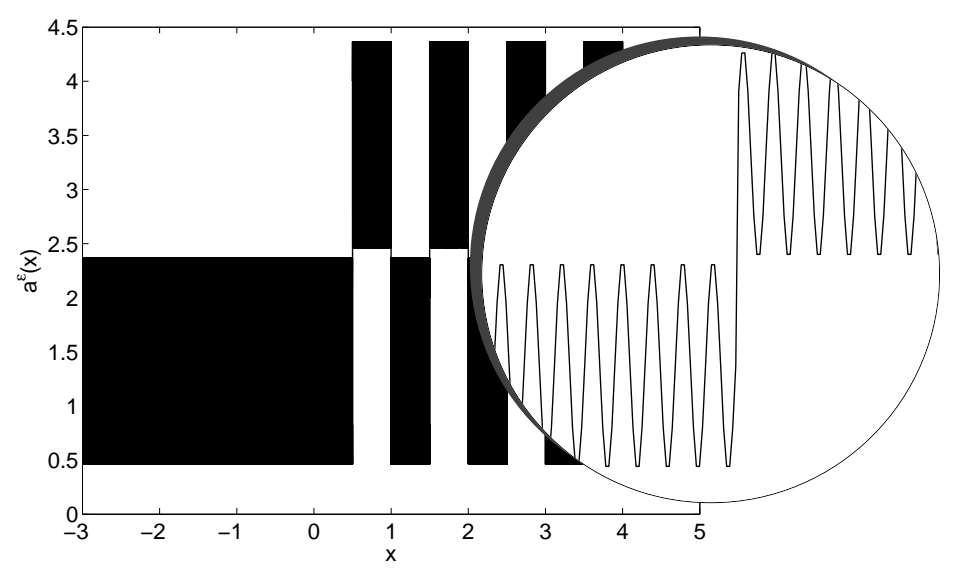

FIG. 5.3. Heterogeneous medium. The squared velocity $a^{\varepsilon}(x)$ defined in (5.4) is shown for $\varepsilon=10^{-3}$, with a partial zoom about $x=4.5$.

We now set $\varepsilon=10^{-3}$ and compute the FE-HMM solution $u^{H}$ on a coarse mesh with $H=10^{-2}$ and time step $\Delta t=10^{-3}$. The sampling domains are of size $\delta=$ $10^{-3}$, each partitioned in equidistant sub-intervals of size $h=10^{-4}$. Again, both the macro and the micro FE spaces consist of continuous piecewise linear functions with $\ell=r=1$. For reference, we also compute $u_{\varepsilon}$ by a fully resolved standard FE solution on a highly refined mesh with $H=10^{-4}$ and $\Delta t=10^{-5}$ to avoid numerical artifacts. Due to the small size of $\varepsilon$ we expect $u_{\varepsilon}$ to be very close to $u_{0}$ (in the $L^{2}$ norm).

Both $u^{H}$ and $u_{\varepsilon}$ are shown in Figure 5.4 and we observe that they essentially coincide (at this scale) until the final time. Hence the FE-HMM is able to capture the main features of the underlying heterogeneous medium at a much smaller computational cost, and in fact independently of $\varepsilon$.

Finally, for the sake of comparison, we also compute the FE solution for an averaged medium $a^{a v}(x)$,

$$
a^{a v}(x)= \begin{cases}2+\sqrt{2} & \text { for } x>0 \text { and } x \in(n-0.5, n) \text { for a } n \in \mathbb{N} \\ \sqrt{2} & \text { elsewhere }\end{cases}
$$

obtained by naive local averaging. The finite element discretization and time step are identical to those of the reference solution. In Figure 5.5, snapshots of the reference solution, $u_{\varepsilon}$, and the solution obtained by simple averaging are shown. As expected, the effective medium $a^{a v}(x)$ does not capture the essential features of the underlying heterogeneous medium and hence the two solutions diverge from each other increasingly with time.

6. Concluding remarks. We have presented a multiscale FE method for wave propagation in heterogeneous media. It is based on a finite element discretization 


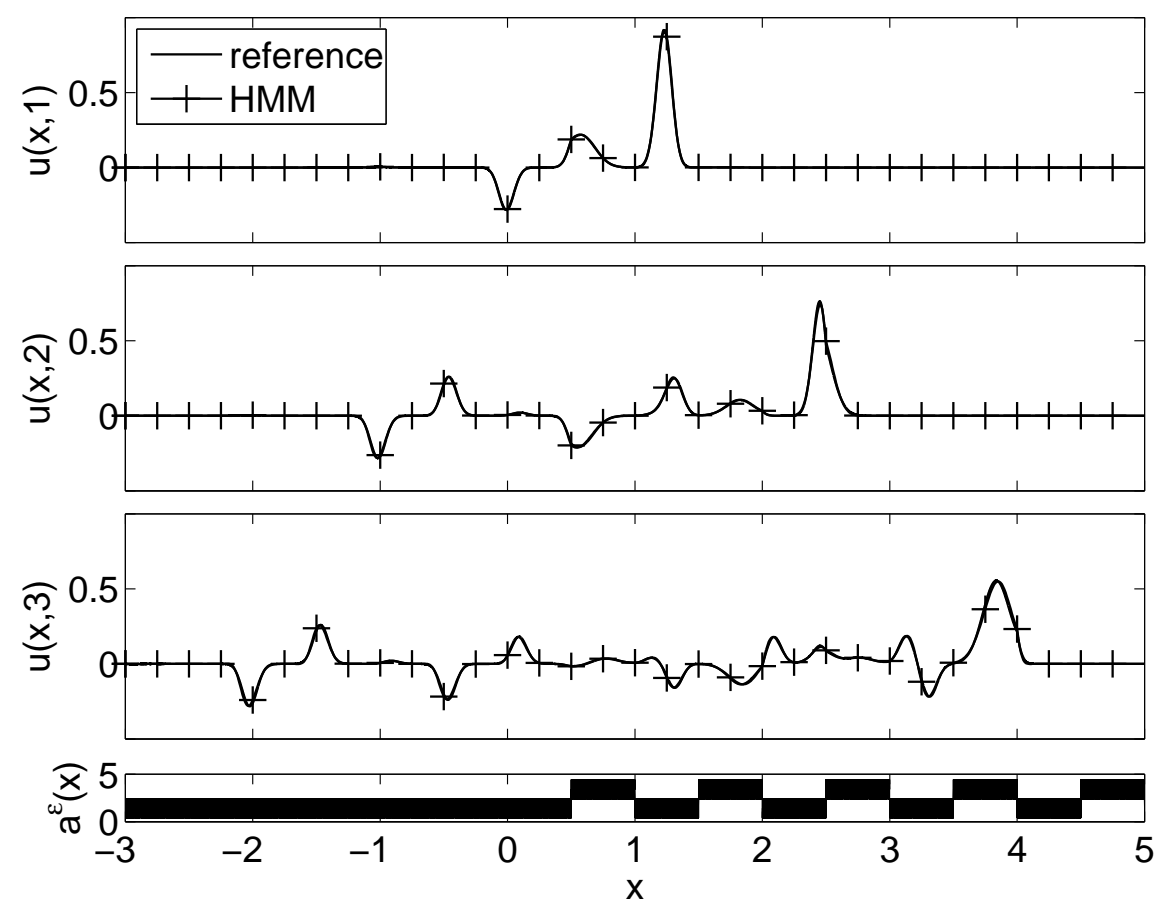

FIG. 5.4. Heterogeneous medium. Snapshots of $u^{H}$ and $u_{\varepsilon}$ at times $t=1,2,3$ of a Gaussian pulse propagating through the medium from Figure 5.3, also depicted here underneath.

of an effective wave equation at the macro scale, whose a priori unknown effective coefficients are computed on sampling domains at the micro scale within each macro finite element. Hence the computational work involved is independent of the highly heterogeneous nature of the medium at the smallest scale $\varepsilon$. Optimal error estimates in the energy norm and the $L^{2}$ norm and convergence to the homogenized solution are proved, when both the macro and the micro scale are refined simultaneously, as corroborated by numerical experiments.

In [20], error estimates of the modeling error with various boundary conditions and oversampling were obtained whereas partial estimates were also derived there for random media. Those results could be used here to estimate the modeling error term for the wave equation in random media.

Because our FE-HMM approach leads to a standard Galerkin finite element formulation at the macro scale, it immediately applies to higher dimensional problems, complex geometry, or high-order discretizations. It also easily generalizes to more complicated second-order hyperbolic equations, such as from electromagnetics or elasticity. Our FE-HMM method and the discontinuous Galerkin HMM [5] can also be combined with discontinuous Galerkin FE methods for the wave equation [23, 24], which provide greater flexibility in the underlying mesh design, waive the need for mass-lumping, and thus lead to inherently parallel fully explicit (local) time stepping schemes [16].

At very long times, waves display a dispersive behavior as they propagate through a highly oscillatory medium, which is not captured by standard homogenization theory. Since our FE-HMM method converges to the classical homogenized solution, it 


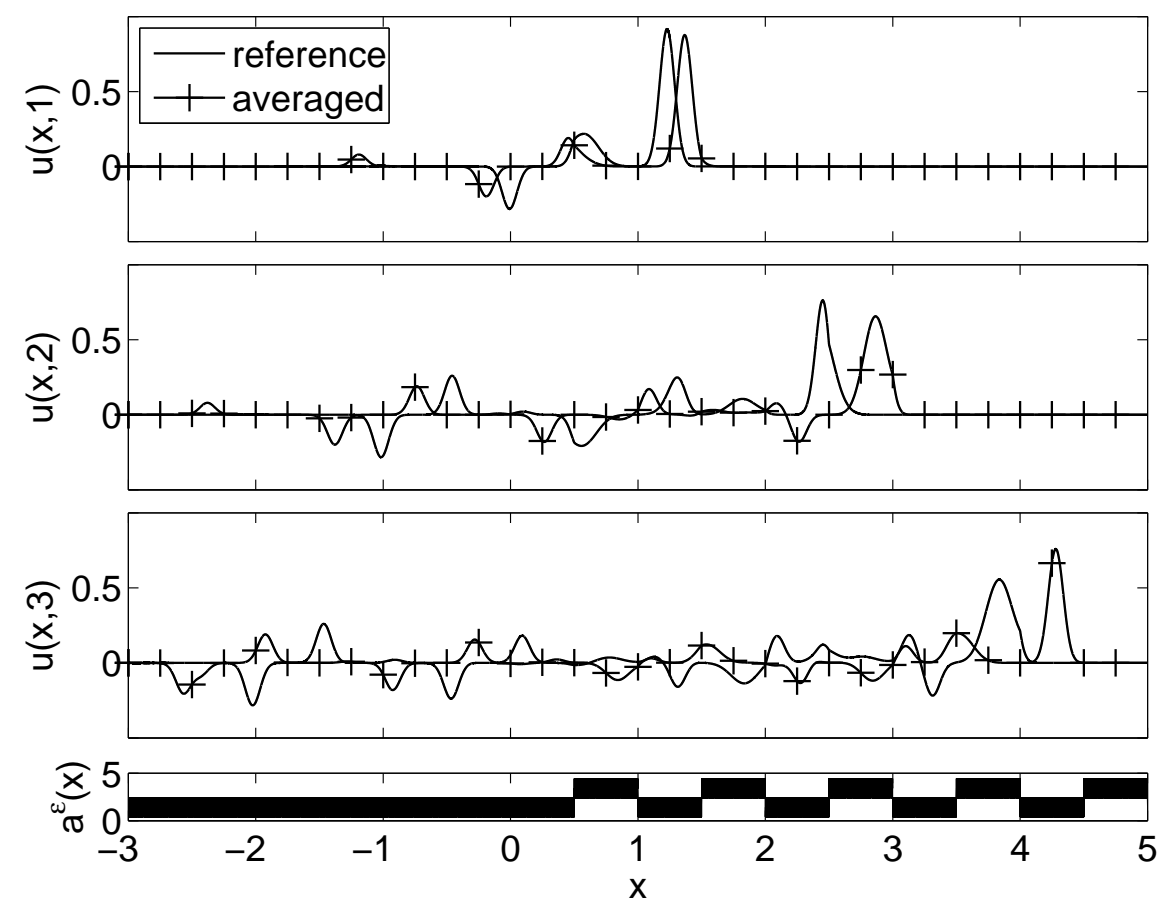

FIG. 5.5. Heterogeneous medium. Snapshots of $u_{\varepsilon}$ and a FE solution for a medium, obtained through a naive averaging procedure, at times $t=1,2,3$ propagating through the medium from Figure 5.3, also depicted here underneath.

cannot capture those long-time effects either, for which higher order corrections are required $[32,14,18]$.

\section{Acknowledgements}

We thank Christian Stohrer for the numerical experiments.

7. Appendix. Here we prove the Strang-like lemma for second-order hyperbolic problems.

Proof of Lemma 4.6. Let $w(t) \in V$ be the elliptic projection of $u_{1}(t)$ with respect to $B_{2}$,

$$
B_{2}(w(t), \chi)=B_{1}\left(u_{1}(t), \chi\right), \quad \forall \chi \in V, \quad t \in[0, T] .
$$

Hence, we immediately have

$$
B_{2}\left(w-u_{1}, \chi\right)=B_{1}\left(u_{1}, \chi\right)-B_{2}\left(u_{1}, \chi\right), \quad \forall \chi \in V .
$$

Now, we let $\chi=w-u_{1}$ and use (4.21) and (4.22) to obtain

$$
\left\|w(t)-u_{1}(t)\right\|_{H^{1}}^{2} \leq C \eta\left\|u_{1}(t)\right\|_{H^{1}}\left\|w(t)-u_{1}(t)\right\|_{H^{1}} .
$$


Henceforth $C$ denotes a generic constant independent of $\eta$. After integrating both sides in time from 0 up to $T$, using Cauchy's inequality [21], and dividing both sides by $\left\|w-u_{1}\right\|_{L^{2}\left(0, T ; H^{1}(\Omega)\right)}$, we obtain

$$
\left\|w-u_{1}\right\|_{L^{2}\left(0, T ; H^{1}(\Omega)\right)} \leq C \eta\left\|u_{1}\right\|_{L^{2}\left(0, T ; H^{1}(\Omega)\right)} .
$$

Since the bilinear forms $B_{1}$ and $B_{2}$ are independent of time, a similar estimate can be derived for $\partial_{t} u_{1}$ and $\partial_{t t} u_{1}$ by differentiating (4.19), (4.20) with respect to time. Thus we have

$$
\left\|\partial_{t}^{k}\left(w-u_{1}\right)\right\|_{L^{2}\left(0, T ; H^{1}(\Omega)\right)} \leq C \eta\left\|\partial_{t}^{k} u_{1}\right\|_{L^{2}\left(0, T ; H^{1}(\Omega)\right)}, \quad k \leq 2 .
$$

We shall now estimate $u_{2}-w$ with $w$ defined by (7.1). Let

$$
\zeta(t)=u_{2}(t)-w(t), \quad t \in[0, T] .
$$

Then from (4.19), (4.20), and (7.1) we observe that

$$
\begin{aligned}
\left(\partial_{t t} \zeta, \chi\right)+B_{2}(\zeta, \chi) & =(F, \chi)-\left(\partial_{t t} w, \chi\right)-B_{2}(w, \chi) \\
& =\left(\partial_{t t} u_{1}, \chi\right)-\left(\partial_{t t} w, \chi\right), \quad \forall \chi \in V
\end{aligned}
$$

Considering for every time $t, 0 \leq t \leq T, \chi(t) \in V$, we thus have

$$
B_{2}(\zeta, \chi)=\left(\partial_{t t}\left(u_{1}-w-\zeta\right), \chi\right), \quad \forall \chi \in V,
$$

which we rewrite as

$$
-\left(\partial_{t} \zeta, \partial_{t} \chi\right)+B_{2}(\zeta, \chi)=\frac{d}{d t}(\beta, \chi)-\left(\phi, \partial_{t} \chi\right)
$$

where

$$
\beta=\partial_{t} u_{1}-\partial_{t} w-\partial_{t} \zeta, \quad \phi=\partial_{t} u_{1}-\partial_{t} w .
$$

We now fix $\xi, 0<\xi \leq T$ and define

$$
\hat{\chi}(t)=\int_{t}^{\xi} \zeta(s) d s, \quad 0 \leq t \leq T .
$$

Hence $\hat{\chi}(t) \in V$ for $0 \leq t \leq T$ and we have

$$
\hat{\chi}(\xi)=0, \quad \partial_{t} \hat{\chi}(t)=-\zeta(t), \quad 0<t \leq T .
$$

We let $\chi=\hat{\chi}$ in (7.6) and integrate both sides with respect to $t$ from 0 to $\xi$. This leads to

$$
\|\zeta(\xi)\|_{L^{2}(\Omega)}^{2}-\|\zeta(0)\|_{L^{2}(\Omega)}^{2}+B_{2}(\hat{\chi}(0), \hat{\chi}(0))=-2(\beta(0), \hat{\chi}(0))+2 \int_{0}^{\xi}(\phi(t), \zeta(t)) d t,
$$

where we have used (7.8) and the symmetry of $B_{2}$. Since

$$
\beta(0)=\partial_{t} u_{1}(0)-\partial_{t} u_{2}(0)=0
$$

and $B_{2}$ satisfies (4.21), we find that

$$
\|\zeta(\xi)\|_{L^{2}(\Omega)}^{2} \leq\|\zeta(0)\|_{L^{2}(\Omega)}^{2}+2\left|\int_{0}^{\xi}(\phi(t), \zeta(t)) d t\right| .
$$


Next, we use the Cauchy-Schwarz inequality and Young's inequality to obtain

$$
\|\zeta(\xi)\|_{L^{2}(\Omega)}^{2} \leq\|\zeta(0)\|_{L^{2}(\Omega)}^{2}+\frac{C}{\delta}\|\phi\|_{L^{2}\left(0, T ; L^{2}(\Omega)\right)}^{2}+C \delta\|\zeta\|_{L^{\infty}\left(0, T ; L^{2}(\Omega)\right)}^{2}, \quad \forall \delta>0 .
$$

Note that the constant $C$ now also depends on $T$. Since the right side is independent of $\xi$, we can take the supremum over $0 \leq \xi \leq T$. For a suitable $\delta$ we thus have

$$
\|\zeta\|_{L^{\infty}\left(0, T ; L^{2}(\Omega)\right)} \leq C\left\{\|\phi\|_{L^{2}\left(0, T ; L^{2}(\Omega)\right)}+\|\zeta(0)\|_{L^{2}(\Omega)}\right\} .
$$

The initial conditions in (4.19), (4.20) and the continuous embedding of $H^{1}\left(0, T ; L^{2}(\Omega)\right)$ into $C^{0}\left([0, T] ; L^{2}(\Omega)\right)$ (Section 5.9.2, [21]) imply

$$
\|\zeta(0)\|_{L^{2}(\Omega)}=\left\|u_{1}(0)-w(0)\right\|_{L^{2}(\Omega)} \leq C\left\{\left\|u_{1}-w\right\|_{L^{2}\left(0, T ; L^{2}(\Omega)\right)}+\left\|\partial_{t}\left(u_{1}-w\right)\right\|_{L^{2}\left(0, T ; L^{2}(\Omega)\right)}\right\} .
$$

Thus from (7.3) and the definition of $\phi$ in (7.7), we can bound the left side of (7.9) as

$$
\begin{aligned}
\|\zeta\|_{L^{\infty}\left(0, T ; L^{2}(\Omega)\right)} & \leq C\left\{\left\|u_{1}-w\right\|_{L^{2}\left(0, T ; L^{2}(\Omega)\right)}+\left\|\partial_{t}\left(u_{1}-w\right)\right\|_{L^{2}\left(0, T ; L^{2}(\Omega)\right)}\right\} \\
& \leq C \eta\left\{\left\|u_{1}\right\|_{L^{2}\left(0, T ; H^{1}(\Omega)\right)}+\left\|\partial_{t} u_{1}\right\|_{L^{2}\left(0, T ; H^{1}(\Omega)\right)}\right\} .
\end{aligned}
$$

Finally, since

$$
\left\|u_{1}-u_{2}\right\|_{L^{\infty}\left(0, T ; L^{2}(\Omega)\right)} \leq\left\|u_{1}-w\right\|_{L^{\infty}\left(0, T ; L^{2}(\Omega)\right)}+\|\zeta\|_{L^{\infty}\left(0, T ; L^{2}(\Omega)\right)}
$$

we conclude again from (7.3) and the continuous embedding of $H^{1}\left(0, T ; L^{2}(\Omega)\right)$ into $C^{0}\left([0, T] ; L^{2}(\Omega)\right)$ that

$$
\left\|u_{1}-u_{2}\right\|_{L^{\infty}\left(0, T ; L^{2}(\Omega)\right)} \leq C \eta\left\{\left\|u_{1}\right\|_{L^{2}\left(0, T ; H^{1}(\Omega)\right)}+\left\|\partial_{t} u_{1}\right\|_{L^{2}\left(0, T ; H^{1}(\Omega)\right)}\right\},
$$

which can be further bounded in terms of $\hat{f}, \hat{g}, F$ and $T$ - see (Section 7.2.3, [21]) for details.

We shall now estimate the "energy norm" of $u_{1}-u_{2}$. Starting from (7.4) and (7.5) above we have

$$
\left(\partial_{t t} \zeta, \chi\right)+B_{2}(\zeta, \chi)=\left(\partial_{t t}\left(u_{1}-w\right), \chi\right), \quad \forall \chi \in V .
$$

We let $\chi(t)=\partial_{t} \zeta(t)$ and use the symmetry of $B_{2}$ to obtain

$$
\frac{1}{2} \frac{d}{d t}\left(\left\|\partial_{t} \zeta\right\|_{L^{2}(\Omega)}^{2}+B_{2}(\zeta, \zeta)\right)=\left(\partial_{t t}\left(u_{1}-w\right), \partial_{t} \zeta\right)
$$

Next, we use the Cauchy-Schwarz inequality, the geometric-arithmetic mean inequality and add $B_{2}(\zeta, \zeta) \geq 0$ to the right side of the resulting expression. This yields

$$
\frac{d}{d t} E(t) \leq C\left\|\partial_{t t}\left(u_{1}-w\right)\right\|_{L^{2}(\Omega)}+E(t),
$$

where the energy $E$ is defined by

$$
E(t)=\left\|\partial_{t} \zeta(t)\right\|_{L^{2}(\Omega)}^{2}+B_{2}(\zeta(t), \zeta(t)) .
$$

By using Gronwall's inequality and the fact that $B_{2}$ satisfies (4.21), we obtain

$$
\begin{aligned}
& \sup _{0 \leq t \leq T}\left(\left\|\partial_{t} \zeta(t)\right\|_{L^{2}(\Omega)}+\|\zeta(t)\|_{H^{1}(\Omega)}\right) \\
& \quad \leq C\left(\left\|\partial_{t} \zeta(0)\right\|_{L^{2}(\Omega)}+\left\|\partial_{t t}\left(u_{1}-w\right)\right\|_{L^{2}\left(0, T ; L^{2}(\Omega)\right)}\|+\| \zeta(0) \|_{H^{1}(\Omega)}\right) .
\end{aligned}
$$


To estimate the third term on the right of (7.11), we use (4.19), (4.20) and the continuous embedding of $H^{1}\left(0, T ; H^{1}(\Omega)\right)$ into $C^{0}\left(0, T ; H^{1}(\Omega)\right)$ [21], which implies

$$
\begin{aligned}
\|\zeta(0)\|_{H^{1}(\Omega)} & =\left\|u_{1}(0)-w(0)\right\|_{H^{1}(\Omega)} \\
& \leq C\left\{\left\|u_{1}-w\right\|_{L^{2}\left(0, T ; H^{1}(\Omega)\right)}+\left\|\partial_{t}\left(u_{1}-w\right)\right\|_{L^{2}\left(0, T ; H^{1}(\Omega)\right)}\right\} .
\end{aligned}
$$

Similarly for the first term on the right of (7.11), we derive the upper bound

$$
\left\|\partial_{t} \zeta(0)\right\|_{L^{2}(\Omega)} \leq C\left\{\left\|\partial_{t}\left(u_{1}-w\right)\right\|_{L^{2}\left(0, T ; L^{2}(\Omega)\right)}+\left\|\partial_{t t}\left(u_{1}-w\right)\right\|_{L^{2}\left(0, T ; L^{2}(\Omega)\right)}\right\} .
$$

Then we estimate the second term on the right of (7.11) together with the terms on the right side of (7.12), (7.13) by using (7.3) with $k=0,1,2$. Hence we can bound the right side of (7.11) by

$$
\sup _{0 \leq t \leq T}\left(\left\|\partial_{t} \zeta(t)\right\|_{L^{2}(\Omega)}+\|\zeta(t)\|_{H^{1}(\Omega)}\right) \leq C \eta \sum_{k=0}^{2}\left\|\partial_{t}^{k} u\right\|_{L^{2}\left(0, T ; H^{1}(\Omega)\right)} .
$$

Since $u_{1}-u_{2}=u_{1}-w-\zeta$ we immediately have

$$
\begin{aligned}
& \left\|\partial_{t}\left(u_{1}-u_{2}\right)\right\|_{L^{2}(\Omega)}+\left\|u_{1}-u_{2}\right\|_{H^{1}(\Omega)} \\
& \leq\left\|\partial_{t} \zeta\right\|_{L^{2}(\Omega)}+\|\zeta\|_{H^{1}(\Omega)}+\left\|\partial_{t}\left(u_{1}-w\right)\right\|_{L^{2}(\Omega)}+\left\|u_{1}-w\right\|_{H^{1}(\Omega)} .
\end{aligned}
$$

To conclude, we estimate $\left\|\partial_{t}\left(u_{1}-w\right)\right\|_{L^{\infty}\left(0, T ; L^{2}(\Omega)\right)}$ and $\left\|u_{1}-w\right\|_{L^{\infty}\left(0, T ; H^{1}(\Omega)\right)}$ as previously from Sobolev embeddings and (7.3), which leads to

$$
\sup _{0 \leq t \leq T}\left(\left\|\partial_{t}\left(u_{1}-u_{2}\right)(t)\right\|_{L^{2}(\Omega)}+\left\|\left(u_{1}-u_{2}\right)(t)\right\|_{H^{1}(\Omega)}\right) \leq C \eta \sum_{k=0}^{2}\left\|\partial_{t}^{k} u\right\|_{L^{2}\left(0, T: H^{1}(\Omega)\right)} .
$$

By assumption the (weak) time derivatives in the sum are bounded. They can also be estimated explicitly by standard energy estimates in terms of $\hat{f}, \hat{g}, F$ and $T$ - see again (Section 7.2.3, [21]) for details.

\section{REFERENCES}

[1] A. Abdulle, On a-priori error analysis of fully discrete heterogeneous multiscale FEM, SIAM Multiscale Model. Simul., 4 (2005), no. 2, 447-459.

[2] A. Abdulle and C. Schwab, Heterogeneous multiscale FEM for diffusion problem on rough surfaces, SIAM Multiscale Model. Simul., 3 (2005), no. 1, 195-220.

[3] A. Abdulle, B. Engquist, Finite element heterogeneous multiscale methods with near optimal computational complexity, SIAM Multiscale Model. Simul. 6 (2007) no. 4, 1059-1084.

[4] A. Abdulle and A. Nonnenmacher, A short and versatile finite element multiscale code for homogenization problems, Comput. Methods Appl. Mech. Engrg. 198 (2009), no. 37-40, 2839-2859.

[5] A. Abdulle, Discontinuous Galerkin finite element heterogeneous multiscale method for elliptic problems with multiple scales, preprint submitted for publication.

[6] A. Abdulle, The finite element heterogeneous multiscale method: a computational strategy for multiscale PDEs, GAKUTO Int. Ser. Math. Sci. Appl., 31 (2009), 135-184.

[7] A. Abdulle, A priori and a posteriori analysis for numerical homogenization: a unified framework, to appear in Higher Education Press, Beijing; World Scientific Publishing Co. Pte. Ltd., Singapore.

[8] A. Abdulle and G. Vilmart, On micro macro errors of the heterogeneous multiscale method for parabolic problems, preprint submitted for publication.

[9] G. A. Baker, Error estimates for finite element methods for second order hyperbolic equations, SIAM J. Numer. Anal., 13 (1976), 564-576. 
[10] G. A. Baker and V. A. Dougalis, The effect of quadrature errors on finite element approximations for second order hyperbolic equations, SIAM J. Numer. Anal., 13 (1976), 577-598.

[11] A. Bensoussan, J.-L. Lions and G. Papanicolaou, Asymptotic Analysis for Periodic Structure, North Holland, Amsterdam, 1978.

[12] L. Bers, F. John, and M. Schechter, Partial differential equations, Lectures in Applied Mathematics, Proceedings of the Summer Seminar, Boulder, CO, 1957.

[13] S. Brahim-Otsmane, G. A. Francfort and F. Murat, Correctors for the homogenization of the wave and heat equations, J. Math. Pures Appl., 71 (1992), 197-231.

[14] W. Chen, J.A. Fish J. A dispersive model for wave propagation in periodic heterogeneous media based on homogenization with multiple spatial and temporal scales. ASME Journal of Applied Mechanics, 68 (2001), 153-161.

[15] P. Ciarlet, The Finite Element Method For Elliptic Problems, North-Holland, Amsterdam, 1978.

[16] J. Diaz and M. J. Grote, Energy conserving explicit local time-stepping for second-order wave equations, SIAM J. Sc. Computing 31 (2009), 1985-2014.

[17] P. Donato and D. Cioranescu, An introduction to Homogenization, Oxford University Press, 1999.

[18] B. Engquist, H. Holst and O. Runborg, Multi-scale methods for wave propagation in heterogeneous media, preprint 2009.

[19] W. E and B. Engquist, The Heterogeneous Multi-Scale Methods, Commun. Math. Sci., 1 (2003), $87-132$.

[20] W. E, P. Ming and P. Zhang, Analysis of the heterogeneous multiscale method for elliptic homogenization problems, J. Amer. Math. Soc. 18 (2005), no. 1, 121-156.

[21] L. C. Evans, Partial Differential Equations, AMS, Providence, RI, 1998.

$[22]$ E. De Giorgi and S. Spagnolo, Sulla convergenza degli integrali dell'energia per operatori ellittici del secondo ordine, Boll. Un. Mat. Ital., 4 (1973), 391-411.

[23] M.J. Grote, A. Schneebeli and D. Schötzau, Discontinuous Galerkin Finite Element Method For The Wave Equation, SIAM J. Num. Analysis 44 (2006), 2408-2431.

[24] M. J. Grote and D. Schötzau, Optimal Error Estimates for the Fully Discrete Interior Penalty DG Method for the Wave Equation, J. Sc. Computing 40 (2009), 257-272.

[25] T-Y. Hou, X-H. Wu and Z. Cai, Convergence of a multiscale finite element method for elliptic problems with rapidly oscillating coefficients, Math. Comp. 68 (1999), no. 227, 913-943.

[26] V. V. Jikov, S.M. Kozlov, and O.A. Oleinik, Homogenization of Differential Operators and Integral Functionals, Springer-Verlag, Berlin,1994.

[27] O.A. Ladyzhenskaya, The boundary value problems of mathematical physics, Applied Mathematical Sciences, 49, Springer-Verlag New York Inc., 1985.

[28] J.-L. Lions and E. Magenes, Probèmes aux limites homogènes et applications, 1, Dunod, Paris, 1968.

[29] F. Murat and L. Tartar, H-convergence, in "Topics in the mathematical modeling of composite materials", A. Cherkaev and R. Kohn Eds., Birkhäuser, Boston(1997), 21-43.

[30] H. Owhadi and L. Zhang, Numerical homogenization of the acoustic wave equations with a continuum of scales, Comput. Methods Appl. Mech. Engrg. 198 (2008), 397-406.

[31] P.A. Raviart, The use of numerical integration in finite element methods for solving parabolic equations, in Miller, J. J. H. (ed.), Topics in Numerical Analysis, Academic Press (1973), $233-264$.

[32] F. Santosa and W. W. Symes, A dispersive effective medium for wave propagation in periodic composites, SIAM J. Appl. Math., 51 (1991 ) 984-1005.

[33] S. Spagnolo, Sulla convergenza di soluzioni di equationi paraboliche ed ellittiche, Ann. Scuola Norm. Sup. Pisa, 22 (1968), 571-597. 


\section{LATEST PREPRINTS}

No. Author: Title

2010-01 Assyr Abdulle, Marcus J. Grote

Finite Element Heterogeneous Multiscale Method for the Wave Equation

2009-06 Marcus J. Grote, Imbo Sim

Efficient PML for the Wave Equation

2009-05 Francesco Amoroso, Evelina Viada

Small Points on Subvarieties of a Torus

2009-04 Evelina Viada

A Functorial Lower Bound for the Essential Minimum of Varieties in a Power of an Elliptic Curve

2009-03 Francesco Amoroso, Evelina Viada

Small Points on Rational Subvarieties of Tori

2009-02 Marcus J. Grote, Teodora Mitkova

Explicit Local Time-Stepping Methods for Maxwell's Equations

2009-01 Marcus J. Grote, Imbo Sim

On Local Nonreflecting Boundary Conditions for Time Dependent Wave

Propagation

2008-05 David Cohen, Xavier Raynaud

Geometric Finite Difference Schemes for the Generalised Hyperelastic-Rod Wave Equation

2008-04 Marcus J. Grote, Dominik Schötzau

Convergence Analysis of a Fully Discrete DG Method for the Wave Equation

2008-03 Matthias Bollhöfer, Marcus J. Grote, Olaf Schenk

Algebraic Multilevel Preconditioner for the Helmholtz Equation in Heterogeneous Media

2008-02 Michael Lenzinger, Ben Schweizer

Two-Phase Flow Equations with Outflow Boundary Conditions in the Hydrophobic-Hydrophilic Case

2008-01 Guido Pezzini

Automorphisms of Wonderful Varieties 